\title{
Preservative-free bimatoprost $0.03 \%$ in patients with primary open-angle glaucoma or ocular hypertension in clinical practice
}

This article was published in the following Dove Press journal:

Clinical Ophthalmology

12 September 2016

Number of times this article has been viewed

\author{
Lutz E Pillunat ${ }^{1}$ \\ Peter Eschstruth ${ }^{2}$ \\ Stefan Häsemeyer ${ }^{3}$ \\ Ulrich Thelen ${ }^{4}$ \\ Christian Foja ${ }^{5}$ \\ Richard Leaback ${ }^{6}$ \\ Stefan Pfennigsdorf ${ }^{7}$ \\ 'Department of Ophthalmology, \\ University Hospital Carl Gustav \\ Carus, Dresden, ${ }^{2}$ Ophthalmology \\ Practice, Kiel, ${ }^{3}$ Eye Center \\ Kraichgau, Wiesloch, ${ }^{4}$ Department of \\ Ophthalmology, University of Münster. \\ Münster, ${ }^{5}$ Ophthalmology Practice, \\ Leipzig, Germany; ${ }^{6}$ Allergan Holdings \\ Ltd., Marlow, UK; ' ${ }^{7}$ Pphthalmology \\ Practice, Polch, Germany
}

Correspondence: Lutz E Pillunat Department of Ophthalmology, University Hospital Carl Gustav Carus Fetscherstrasse 74, 01307 Dresden, Germany

Tel +4935 |458338|

Email augendirektion@uniklinikumdresden.de
Background: Intraocular pressure (IOP)-lowering medications for primary open-angle glaucoma and ocular hypertension commonly contain preservatives that can cause ocular surface damage in many patients. The purpose of this study was to evaluate the efficacy and tolerability of, and compliance to, preservative-free $(\mathrm{PF})$ bimatoprost $0.03 \%$ in patients with primary openangle glaucoma or ocular hypertension ( $\mathrm{IOP} \geq 18 \mathrm{mmHg}$ ) in a clinical practice setting.

Methods: This open-label study observed patients who were switched to PF bimatoprost $0.03 \%$ for medical reasons. IOP was measured at baseline and $\sim 12$ weeks later at the final visit, and the change in IOP was calculated. Tolerability and continuation of therapy were assessed at two follow-up visits.

Results: A total of 1,830 patients were included in the study, and complete IOP data were available for 1,543 patients. Mean IOP was reduced by $23 \%$ from $21.64 \mathrm{mmHg}$ to $16.59 \mathrm{mmHg}$ $(P<0.0001)$. In subgroup analyses, the mean IOP was significantly reduced compared with baseline, regardless of prior therapy, including those previously treated with PF monotherapy. A total of $85.7 \%$ of physicians reported the IOP-lowering efficacy of PF bimatoprost $0.03 \%$ to be as expected or better than expected. Adverse events (AEs) were experienced by $5.7 \%$ of patients, and there were no serious AEs reported. The most common AEs were eye irritation $(1.7 \%)$ and hyperemia (1.4\%). Physician-reported treatment compliance was reported as better than $(48.7 \%)$ or equal to $(43.6 \%)$ prior treatment in most patients. Most patients $(82 \%)$ were expected to continue PF bimatoprost $0.03 \%$ after the end of the study.

Conclusion: This observational study showed that, in clinical practice, switching to PF bimatoprost $0.03 \%$ was associated with a significant IOP reduction from baseline. There was a low AE rate. PF bimatoprost $0.03 \%$ may, therefore, be an effective treatment option for patients who are intolerant of preservatives or have an inadequate response to prior IOP-lowering treatments.

Keywords: bimatoprost $0.03 \%$, intraocular pressure, prostaglandin, preservative free, benzalkonium chloride free

\section{Introduction}

Glaucoma is the second leading cause of blindness and a leading cause of visual impairment, which can affect walking and mobility. ${ }^{1,2}$ Glaucoma-associated visual impairment increases the risk of falls, ${ }^{3}$ leads to avoidance of difficult situations (eg, night driving), ${ }^{4}$ and has a negative effect on patients' quality of life. ${ }^{1}$ In 2013 , the worldwide prevalence of glaucoma was 3.54\% with an estimated 64.3 million people affected by the disease. This number is expected to rise by $18.2 \%$ in 2020 and by $73.8 \%$ in 2040, to 76.0 and 111.8 million people worldwide, respectively. 
In patients with primary open-angle glaucoma (POAG) or ocular hypertension (OHT), the main goal of treatment is to preserve the visual function and associated quality of life by lowering intraocular pressure (IOP). ${ }^{2}$ Prostaglandin analog (PGA) monotherapy is a first-line treatment option owing to its IOP-lowering efficacy, lack of systemic side effects, and once-daily dosing requirement. ${ }^{2}$ A network meta-analysis demonstrated that bimatoprost $0.03 \%$ (Lumigan $0.03 \%$; Allergan, Inc., Irvine, CA, USA) is the PGA with the greatest overall ability to lower the mean IOP vs placebo. ${ }^{5}$

Glaucoma therapies, including PGAs, are frequently formulated with preservatives. Preserved glaucoma therapies have been shown to have cytotoxic effects on the tissues of the ocular surface, ${ }^{6}$ and preservatives may contribute to inflammation, exacerbating preexisting ocular surface disease such as dry eye disease and meibomian gland dysfunction. ${ }^{7}$ There is a high prevalence of ocular surface disease in glaucoma patients, which is associated with glaucoma severity and duration and the number of IOP-lowering medications that a patient is taking. ${ }^{8}$ Among those patients who switched from preserved PGA monotherapy to preservative-free (PF) tafluprost, the number of patients with moderate or severe hyperemia fell significantly from baseline to the end of study. ${ }^{9}$

To address the need for PF glaucoma therapies for patients who are intolerant to preservatives, $\mathrm{PF}$ bimatoprost $0.03 \%$ was developed with the aim of delivering the IOP-lowering efficacy of bimatoprost $0.03 \%$, without the potential for long-term tolerability problems associated with preservatives. A randomized, double-blind clinical trial assessed the IOP-lowering efficacy and tolerability profile of $\mathrm{PF}$ bimatoprost $0.03 \%$ compared with preserved bimatoprost $0.03 \% .{ }^{10}$ Over 12 weeks of treatment, PF bimatoprost $0.03 \%$ was found to be noninferior to preserved bimatoprost $0.03 \%$ in terms of IOP-lowering efficacy and significantly decreased IOP by $5.93-7.49 \mathrm{mmHg}$ from baseline. The efficacy and tolerability of PF bimatoprost $0.03 \%$ was equivalent to that of preserved bimatoprost $0.03 \%$.

The objective of this study was to evaluate the efficacy in terms of change from baseline in IOP, tolerability, and continuation of the use of PF bimatoprost $0.03 \%$ monotherapy in patients with POAG or OHT who switched from a prior IOP-lowering therapy for medical reasons, in a real-life clinical practice setting.

\section{Methods}

\section{Study design}

This noninterventional, open-label, prospective study (NCT01853085) collected data on the efficacy and tolerability of PF bimatoprost $0.03 \%$ in a routine clinical practice setting. The study was approved by an independent ethics committee (Landesärztekammer Baden-Württemberg, Stuttgart, Germany) and complied with the principles of Good Clinical Practice and the Declaration of Helsinki. All patients provided written informed consent.

This study was conducted in 312 centers across Germany. Patients were required to have a diagnosis of POAG or OHT with an IOP of $\geq 18 \mathrm{mmHg}$ in at least one eye. Patients were required to have been previously prescribed topical IOPlowering therapy but with insufficient IOP control prior to the start of the study. There were no formal exclusion criteria for this study, and patients were prescribed PF bimatoprost $0.03 \%$ at the discretion of their treating physician, following their usual clinical practice. Treatment was guided by the Summary of Product Characteristics, which recommends one drop of PF bimatoprost $0.03 \%$ once daily to each affected eye. ${ }^{11}$ No washout period between treatments was required. Patients were followed for up to 12 weeks, and IOP measurements were made using a Goldmann tonometer. At the first visit, patients were assessed for baseline characteristics prior to commencing PF bimatoprost $0.03 \%$, including IOP value and ocular symptoms. Following the commencement of PF bimatoprost $0.03 \%$ treatment, assessments were made at two further visits: visit 2 at $2-4$ weeks and visit 3 at $8-12$ weeks. Follow-up visit assessments included IOP values, adverse events (AEs), physician assessment of efficacy, discontinuation during the observational period, continuation of therapy beyond the end of the study period, and compliance compared with previous therapy. Physician and patient assessment of tolerability were evaluated using the standard case report form at each follow-up visit. The primary efficacy variable was the mean change in IOP from baseline to final visit. The secondary objectives were to evaluate the tolerability and continued use of PF bimatoprost $0.03 \%$ in a routine clinical setting.

\section{Analysis}

Data entry and analysis were carried out using the software of Syneed Medidata GmbH (Konstanz, Germany) and the statistical software package SAS (SAS Institute Inc., Cary, NC, USA). Medications were analyzed on the basis of original entries and international nonproprietary names. Data analyses were performed descriptively, and this included preparation of data listings and summary statistics (extreme values, interquartile section, mean and median values, and $\mathrm{SD})$ or frequency distribution tables, as appropriate for each item. Data for demographic characteristics at baseline, efficacy and tolerability assessments, discontinuation and continuation of therapy, and compliance compared with prior therapy were provided for all patients treated with $\mathrm{PF}$ bimatoprost $0.03 \%(n=1,676)$. AE data were provided for the 
safety population, consisting of all 1,830 patients enrolled in the study. Patients with complete data $(n=1,543)$ were included for statistical analysis of the change in IOP from baseline to final visit. These data were compared using a paired $t$-test at the two-sided 5\% level. Subgroup analysis by prior IOP-lowering monotherapy was also performed.

\section{Results}

A total of 1,830 patients were included in the study, and the PF bimatoprost $0.03 \%$ monitoring period took place between April 2013 and March 2014. Baseline data were available for 1,676 patients who were switched to PF bimatoprost $0.03 \%$ and who met the inclusion criterion of a baseline IOP of $\geq 18 \mathrm{mmHg}$ in at least one eye.

\section{Patient demographics}

Baseline patient characteristics are presented in Table 1. Most patients (96.7\%) had previously been treated with an IOP-lowering therapy, and in the majority of these patients $(62.8 \%)$, the prescribed IOP-lowering therapy contained preservatives. Prior therapies reported in $\geq 2 \%$ of patients are shown in Figure 1. Tafluprost and latanoprost were the most commonly reported prior therapies. Of those patients previously treated, $37.2 \%$ switched from a PF prostaglandin monotherapy to PF bimatoprost $0.03 \%$ monotherapy.

\section{Reasons for switching to PF bimatoprost $0.03 \%$ therapy}

The most frequently reported reason for switching from a prior IOP-lowering therapy to PF bimatoprost $0.03 \%$ was inadequate IOP control, which was reported in $73.9 \%$ of

Table I Patient demographic data at baseline

\begin{tabular}{ll}
\hline Patient characteristics & Patients \\
\hline Median age years, $\mathrm{n}$ (interquartile range) & $68(59.0-75.0)^{\mathrm{a}}$ \\
Male, $\mathrm{n}(\%)$ & $668(39.9)^{\mathrm{b}}$ \\
Female, $\mathrm{n}(\%)$ & $\mathrm{I}, 001(59.7)^{\mathrm{b}}$ \\
Median time since first diagnosis, years & $2.9(0.9-7.7)^{\mathrm{a}}$ \\
(interquartile range) & \\
Diagnosis, $\mathrm{n}(\%)$ & \\
OHT & $416(24.8)^{\mathrm{b}}$ \\
OAG & $1,419(84.7)^{\mathrm{b}}$ \\
$\quad$ Pseudoexfoliation glaucoma & $93(6.6)^{\mathrm{c}}$ \\
Pigmentary glaucoma & $13(0.9)^{\mathrm{c}}$ \\
Previous IOP-lowering therapy, $\mathrm{n}$ & $\mathrm{I}, 620$ \\
Preserved & $1,017(62.8)^{\mathrm{d}}$ \\
Preservative free & $603(37.2)^{\mathrm{d}}$ \\
\hline
\end{tabular}

Notes: All patients treated with PF bimatoprost $0.03 \%$ had an IOP of $\geq 18 \mathrm{mmHg}$ in at least one eye at baseline $(n=1,676)$. Percentages may total more or less than 100

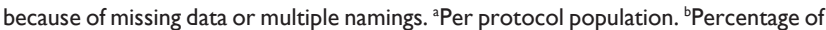
the per protocol population $(n=1,676)$. Percentage of patients with OAG $(n=1,419)$. ${ }^{d}$ Percentage of patients on prior therapy $(n=I, 620)$.

Abbreviations: IOP, intraocular pressure; OAG, open-angle glaucoma; OHT, ocular hypertension; PF, preservative free. patients. Insufficient tolerability, evidence of glaucomatous disease progression, and lack of compliance with prior therapy were reported in $37.4 \%, 18.8 \%$, and $12.3 \%$ of patients, respectively.

\section{Effect on IOP}

For patients with complete data, switching from a prior IOP-lowering therapy to PF bimatoprost $0.03 \%$ significantly decreased the mean IOP by $23.4 \%$ compared with baseline (Figure 2).

For patients on any prior IOP-lowering monotherapy, the mean \pm SD IOP was $21.43 \pm 2.81 \mathrm{mmHg}$ at baseline and $16.60 \pm 2.61 \mathrm{mmHg}$ at the final visit, which was a reduction of $22.5 \%(P<0.0001$ [complete data on IOP were available for 759 patients on any prior IOP-lowering monotherapy]). For all other prior IOP-lowering therapy subgroups analyzed, PF bimatoprost $0.03 \%$ monotherapy significantly decreased the mean IOP compared with baseline (Figure 3). At the final study visit, the majority of physicians stated that the IOPlowering efficacy of PF bimatoprost $0.03 \%$ was either better than expected $(36.8 \%)$ or expected $(48.9 \%)$.

\section{AEs and tolerability}

Data on tolerability were available for 1,830 patients. A total of 105 patients $(5.7 \%$ ) reported AEs (Table 2). The most common AEs reported were eye irritation (1.7\%) and hyperemia $(1.4 \%)$. No serious AEs were reported. One fatal outcome was recorded. In addition to POAG, this patient also suffered from arterial hypertension and coronary artery disease. According to the attending physician, there was no causal relationship between the fatal outcome and PF bimatoprost $0.03 \%$ therapy. The tolerability of PF bimatoprost $0.03 \%$ was rated as being either "very good" or "good" by $87.3 \%$ of patients and $89.7 \%$ of physicians. In 1,676 patients with an IOP of $\geq 18 \mathrm{mmHg}$ at baseline who received PF bimatoprost $0.03 \%$, a minority of patients $(7.5 \%)$ discontinued PF bimatoprost $0.03 \%$ therapy (data on discontinuation were missing for $3.3 \%$ of patients). The most common reasons for discontinuation are shown in Table 3. Physician-rated patient compliance with bimatoprost $0.03 \%$ was better than previous therapy in $48.7 \%$ of patients, was equal in $43.6 \%$ of patients, and was worse in $2.5 \%$ of patients.

\section{Discussion}

In this observational, open-label study, a PF formulation of bimatoprost $0.03 \%$ was assessed for mean IOP-lowering efficacy in patients with uncontrolled IOP, despite the use of IOP-lowering agents. Switching from a prior IOP-lowering agent to PF bimatoprost $0.03 \%$ was associated with a mean 


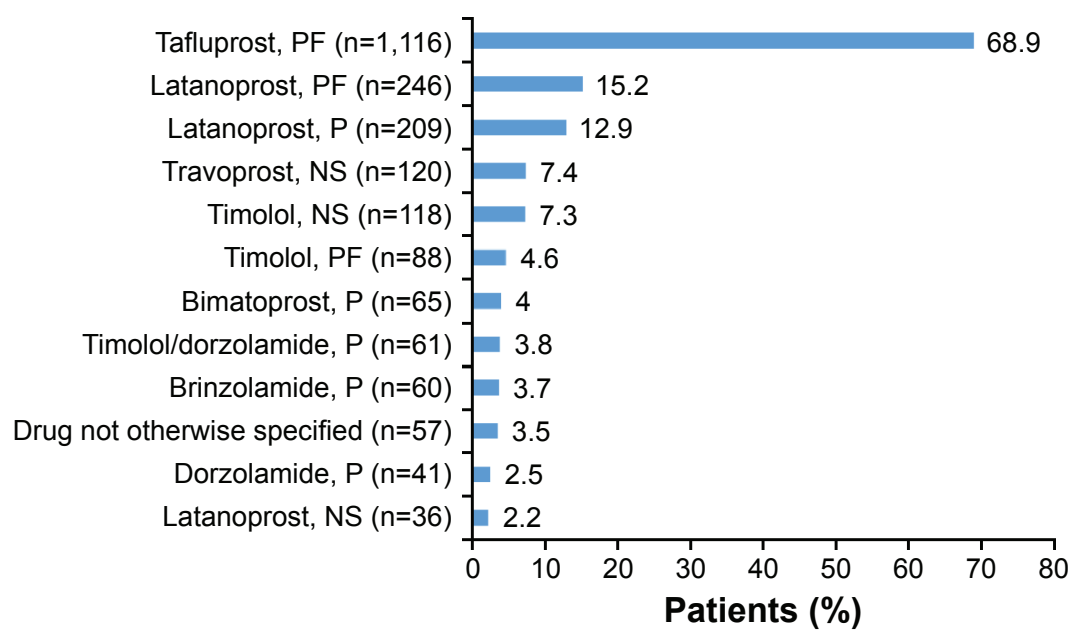

Figure I Prior intraocular pressure-lowering therapy reported in $\geq 2 \%$ of patients.

Notes: Medications as listed by treating physician. The figure includes patients receiving both monotherapy and combination therapy $(\mathrm{n}=\mathrm{I}, 620)$.

Abbreviations: NS, not specified; P, preserved; PF, preservative free.

IOP reduction of $23.4 \%$ after 12 weeks of treatment. Compared with other classes of IOP-lowering agents, including $\beta$-blockers, $\alpha 2$ adrenergic agents, and carbonic anhydrase inhibitors, PGAs are among the most effective treatment in reducing IOP in POAG and OHT. ${ }^{12}$ In a meta-analysis of eight clinical trials, bimatoprost $0.03 \%$ demonstrated significantly greater IOP-lowering efficacy compared with latanoprost and travoprost. The improved IOP-lowering efficacy of bimatoprost $0.03 \%$ was stable throughout the day, as measured at four time points. ${ }^{13}$ A network metaanalysis also ranked bimatoprost $0.03 \%$ highest according to IOP-lowering effect compared with other glaucoma

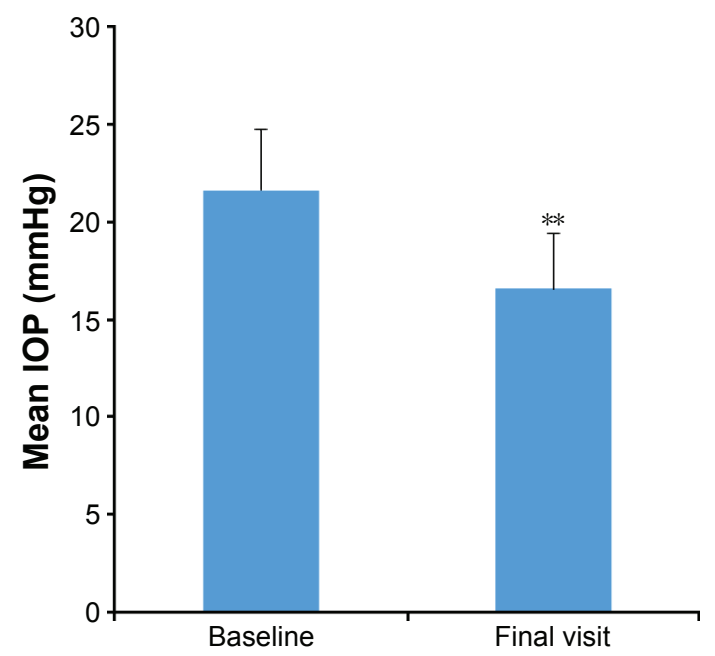

Figure 2 Overall mean \pm SD IOP in patients who switched from a prior IOPlowering therapy to preservative-free bimatoprost $0.03 \%$ monotherapy.

Notes: Data reported for patients with complete data $(n=1,543$ at both visits). $* * P<0.000$ I.

Abbreviation: IOP, intraocular pressure. therapies, including travoprost, latanoprost, and timolol. ${ }^{5}$ It should be noted that no head-to-head studies have compared the efficacy and tolerability of PF bimatoprost $0.03 \%$ with other types of PF or preserved IOP-lowering agents. Previously, PF bimatoprost $0.03 \%$ was shown to be equivalent to preserved bimatoprost $0.03 \%$ in terms of IOP-lowering efficacy and tolerability. ${ }^{14}$

The negative effects of preservatives on the ocular surface are becoming increasingly apparent. ${ }^{8}$ The detergent properties of benzalkonium chloride (BAC) may destabilize the lipid layer of the tear film, permitting excessive aqueous evaporation and resulting in hyperosmolarity. This is further supported by a study in a three-dimensional reconstructed corneal epithelia model, where BAC-containing glaucoma therapies induced significant corneal epithelial deterioration compared with polyquad-preserved formulations. ${ }^{15}$ However, an open-label surveillance study found that switching from BAC- to polyquad-preserved tavoprost $0.004 \%$ was associated with an average $2.92 \mathrm{mmHg}$ increase in IOP, suggesting that polyquad may be less able to promote drug absorption, thereby affecting the IOP-lowering ability. ${ }^{16}$

Increased tear osmolarity has been found in patients with glaucoma and OHT, correlating with Ocular Surface Disease Index questionnaire score and tear film break-up time, a measure of the stability of the tear film. Furthermore, a correlation has been found between the number of instillations of preserved eye drops and ocular surface disease symptoms, as well as tear osmolarity. ${ }^{17}$ Dry eye disease is an ocular surface disease where tear hyperosmolarity is a central driver, inducing apoptosis of corneal epithelial cells and ocular inflammation. ${ }^{17-19}$ 


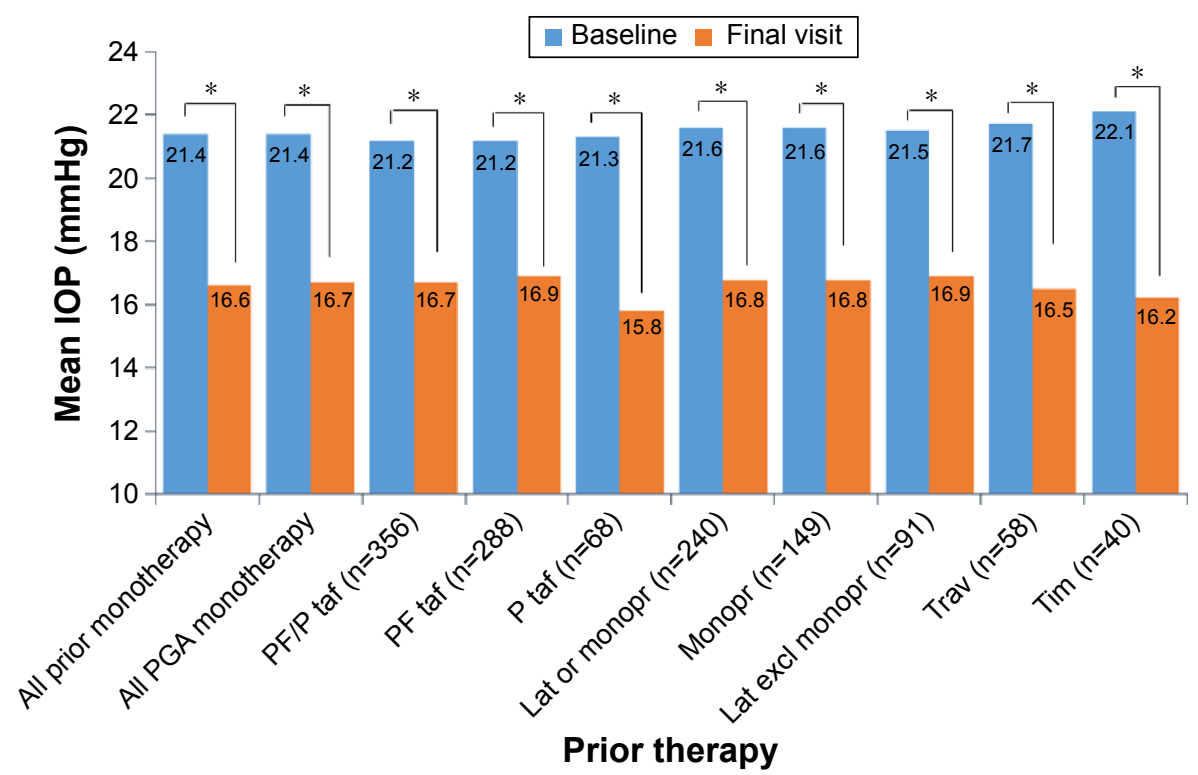

Figure 3 Mean \pm SD IOP in patients switched from a prior IOP-lowering monotherapy to PF bimatoprost $0.03 \%$ monotherapy

Notes: Data reported for patients with complete data. $* P<0.000$ I.

Abbreviations: excl, excluding; IOP, intraocular pressure; lat, latanoprost; monopr, monoprost; P, preserved; PF, preservative free; PGA, prostaglandin analog; taf, tafluprost; tim, timolol; trav, travoprost.

Importantly, the current study demonstrated significant IOP-lowering efficacy of PF bimatoprost $0.03 \%$ in patients whose IOP had been insufficiently controlled by prior PF prostaglandins, including tafluprost, latanoprost, and travoprost. This suggests that PF bimatoprost $0.03 \%$ may be a suitable option in patients requiring PF glaucoma therapies and in whom prior PF treatments have failed to sufficiently lower IOP.

PF PGA therapy was well tolerated, and $>90 \%$ of patients did not report any AEs throughout the observational period. In epidemiological studies where patients were switched from preserved to PF IOP-lowering medications (PF PGAs were unavailable at the time of the study), a significant reduction in the prevalence of ocular pain or discomfort was found. ${ }^{20,21}$ More recently, a study has investigated switching from a preserved to a PF PGA, latanoprost, and found a similar trend in ocular signs and symptoms. ${ }^{9}$ The majority of patients in this study reported an improvement in burning, foreign body

Table 2 Incidence of adverse events reported in $\geq 0.5 \%$ of patients

\begin{tabular}{ll}
\hline Adverse event & Patients, $\mathbf{n}(\%)^{\mathbf{a}}$ \\
\hline Eye irritation & $32(1.7)$ \\
Hyperemia & $26(1.4)$ \\
Erythema of the eyelid & $19(1.0)$ \\
Eye pain & $17(0.9)$ \\
Foreign body sensation & $15(0.8)$ \\
Eye pruritus & $14(0.8)$ \\
\hline
\end{tabular}

Note: aPercentage of the safety population $(n=1,830)$. sensation, itching, irritation, stinging, tearing, and dryness following the change in PF medication. Therefore, it may be of interest to investigate the impact of switching from a preserved IOP-lowering therapy to a PF bimatoprost $0.03 \%$ therapy on ocular surface symptoms in patients with preexisting ocular surface disorders in a future study.

PF bimatoprost $0.03 \%$ was associated with high rates of compliance compared with prior therapy. Physician assessment of patient compliance was reported as better or equal in $93.0 \%$ of patients, and the majority of patients $(82.0 \%)$ were expected to continue PF bimatoprost $0.03 \%$ therapy after the study concluded.

\section{Study limitations}

As this was an open-label, observational study, there are limitations that may affect the interpretation of the results.

Table 3 Reasons for discontinuing PF bimatoprost 0.03\% therapy

\begin{tabular}{ll}
\hline Reason & Patients, $\mathbf{n}(\%)$ \\
\hline Unacceptable ocular tolerability & $64(31.1)$ \\
Insufficient IOP control & $61(29.6)$ \\
Patients lost to follow-up & $22(10.7)$ \\
Patients' decision to withdraw from study & $17(8.3)$ \\
Physicians decision to withdraw patient from study & $14(6.8)$ \\
Other reason & $42(20.4)$ \\
\hline
\end{tabular}

Notes: All patients treated with PF bimatoprost $0.03 \%$ had an IOP of $\geq 18 \mathrm{mmHg}$ in at least one eye at baseline $(n=1,676)$. Percentages may total $<100$ because of missing data.

Abbreviations: IOP, intraocular pressure; PF, preservative free. 
A selected patient population was included in the study at the treating physician's discretion, potentially leading to selection bias, and patients with missing data were not analyzed. A standardized procedure for data recording was not used, meaning that factors such as the time of day that the measurement was taken may affect the results. Data on pseudoexfoliation and pigmentary glaucoma were not requested by the protocol but, owing to the observational nature of the study, some such data were provided. Additionally, the design was uncontrolled, and there was no washout period between any prior therapy and beginning PF bimatoprost $0.03 \%$, meaning that outcomes may be affected by residual effects of prior treatments. The short-term duration of the study was inadequate for the evaluation of long-term safety and efficacy with PF bimatoprost $0.03 \%$. Furthermore, as efficacy was compared with baseline measurements and not a control group, it was not possible to compare the IOP-lowering efficacy of PF bimatoprost $0.03 \%$ with that of other IOP-lowering therapies or to control the natural variation in IOP measurements that were unrelated to treatment.

A controlled, double-masked, crossover study would address some of these limitations and allow further assessment of the comparative efficacy of PF bimatoprost $0.03 \%$ with other preserved or PF IOP-lowering therapies.

\section{Conclusion}

Taken together, the results of this study suggest that PF bimatoprost $0.03 \%$ may be an appropriate IOP-lowering treatment for glaucoma patients who are sensitive to preservatives or for those who have an inadequate response to other IOP-lowering treatments. Switching to PF bimatoprost $0.03 \%$ was associated with a mean IOP reduction of $23 \%$ from $21.64 \mathrm{mmHg}$ to $16.59 \mathrm{mmHg}(P<0.0001)$. Physicianreported treatment compliance was reported as better than $(48.7 \%)$ or equal to $(43.6 \%)$ prior treatment in most patients. There was a low rate of AEs (5.7\%) and no serious adverse drug reactions. Most patients (82\%) were expected to continue PF bimatoprost $0.03 \%$ after the end of the study.

\section{Acknowledgments}

This observational study was funded by Allergan Ltd. The authors thank Newton Healthcare Communications for writing and editorial support, funded by Allergan Ltd. The abstract of this article was presented at COPHy 2015 6th World Congress in Sorrento, Italy, on March 26-29, 2015, as an abstract presentation with initial findings.

\section{Disclosure}

LEP gives lectures for Allergan and Santen and is on the advisory board for Santen. PE gives lectures for Allergan, Novartis, and AstraZeneca. SH, UT, and SP give lectures for Allergan. CF gives lectures for Allergan, Novartis, Pfizer, and Bayer. RL is employed by Allergan. The authors report no other conflicts of interest in this work.

\section{References}

1. Freeman EE, Munoz B, West SK, Jampel HD, Friedman DS. Glaucoma and quality of life: the Salisbury eye evaluation. Ophthalmology. 2008; 115(2):233-238.

2. European Glaucoma Society. Terminology and Guidelines for Glaucoma. 4th ed. Savona, Italy: PubliComm; 2014.

3. Haymes SA, LeBlanc RP, Nicolela MT, Chiasson LA, Chauhan BC. Risk of falls and motor vehicle collisions in glaucoma. Invest Ophthalmol Vis Sci. 2007;48(3):1149-1155.

4. Ramulu PY, West SK, Munoz B, Jampel HD, Friedman DS. Driving cessation and driving limitation in glaucoma: the Salisbury eye evaluation project. Ophthalmology. 2009;116(10):1846-1853.

5. van der Valk R, Webers CA, Lumley T, Hendrikse F, Prins MH, Schouten JS. A network meta-analysis combined direct and indirect comparisons between glaucoma drugs to rank effectiveness in lowering intraocular pressure. J Clin Epidemiol. 2009;62(12):1279-1283.

6. Baudouin C, Labbe A, Liang H, Pauly A, Brignole-Baudouin F. Preservatives in eyedrops: the good, the bad and the ugly. Prog Retin Eye Res. 2010;29(4):312-334.

7. International Dry Eye Workshop. Report of the international dry eye workshop (DEWS). Ocul Surf. 2007;5(2):61-204.

8. Stalmans I, Megevand SG, Cordeiro F, et al. Preservative-free treatment in glaucoma: who, when, and why. Eur J Ophthalmol. 2013;23(4):518-525.

9. Hommer A, Kimmich F. Switching patients from preserved prostaglandin-analog monotherapy to preservative-free tafluprost. Clin Ophthalmol. 2011;5:623-631.

10. Day DG, Walters TR, Schwartz GF, et al. Bimatoprost $0.03 \%$ preservative-free ophthalmic solution versus bimatoprost $0.03 \%$ ophthalmic solution (Lumigan) for glaucoma or ocular hypertension: a 12-week, randomised, double-masked trial. Br J Ophthalmol. 2013;97(8):989-993.

11. Allergan Ltd [webpage on the Internet]. Lumigan $0.3 \mathrm{mg} / \mathrm{ml}$ Summary of Product Characteristics. electronic Medicines Compendium (eMC); 2014. Available from: http://emc.medicines.org.uk/medicine/8925/ SPC/Lumigan/. Accessed August 16, 2015.

12. van der Valk V, Webers CA, Schouten JS, Zeegers MP, Hendrikse F, Prins MH. Intraocular pressure-lowering effects of all commonly used glaucoma drugs: a meta-analysis of randomized clinical trials. Ophthalmology. 2005;112(7):1177-1185.

13. Aptel F, Cucherat M, Denis P. Efficacy and tolerability of prostaglandin analogs: a meta-analysis of randomized controlled clinical trials. J Glaucoma. 2008;17(8):667-673.

14. Day D, Walters TR, Schwartz G, et al. Bimatoprost $0.03 \%$ preservative-free ophthalmic solution versus bimatoprost $0.03 \%$ ophthalmic solution (Lumigan) for glaucoma or ocular hypertension: a 12-week, double-masked trial. Poster presented at: European Glaucoma Society Congress; 2012; Copenhagen, Denmark.

15. Liang H, Pauly A, Riancho L, Baudouin C, Brignole-Baudouin F. Toxicological evaluation of preservative-containing and preservativefree prostaglandin analogs on the 3D-reconstituted corneal epithelium (SkinEthic) system. Br J Ophthalmol. 2011;95(6):869-875.

16. Eschstruth P, Pfennigsdorf S. Comparison of the IOP-lowering efficacy of BAK-preserved Travatan versus polyquad-preserved Travatan. In: German Ophthalmological Society Congress. Berlin; 2013: Abstract PSa09-PSa10. 
17. Labbe A, Terry O, Brasnu E, Van WC, Baudouin C. Tear film osmolarity in patients treated for glaucoma or ocular hypertension. Cornea. 2012; 31(9):994-999.

18. International Dry Eye Workshop. The definition and classification of dry eye disease: report of the Definition and Classification Subcommittee of the International Dry Eye WorkShop (2007). Ocul Surf. 2007; 5(2):75-92.

19. Tomlinson A, Khanal S, Ramaesh K, Diaper C, McFadyen A. Tear film osmolarity: determination of a referent for dry eye diagnosis. Invest Ophthalmol Vis Sci. 2006;47(10):4309-4315.
20. Jaenen N, Baudouin C, Pouliquen P, Manni G, Figueiredo A, Zeyen T. Ocular symptoms and signs with preserved and preservative-free glaucoma medications. Eur J Ophthalmol. 2007;17(3):341-349.

21. Pisella PJ, Pouliquen P, Baudouin C. Prevalence of ocular symptoms and signs with preserved and preservative free glaucoma medication. Br J Ophthalmol. 2002;86(4):418-423.

\section{Publish your work in this journal}

Clinical Ophthalmology is an international, peer-reviewed journal covering all subspecialties within ophthalmology. Key topics include: Optometry; Visual science; Pharmacology and drug therapy in eye diseases; Basic Sciences; Primary and Secondary eye care; Patient Safety and Quality of Care Improvements. This journal is indexed on

Submit your manuscript here: http://www.dovepress.com/clinical-ophthalmology-journal

\section{Dovepress}

PubMed Central and CAS, and is the official journal of The Society of Clinical Ophthalmology (SCO). The manuscript management system is completely online and includes a very quick and fair peer-review system, which is all easy to use. Visit http://www.dovepress.com/ testimonials.php to read real quotes from published authors. 